\title{
Research Paper: Hippocampal Astrocyte Response to Melatonin Following Neural Damage Induction in Rats
}

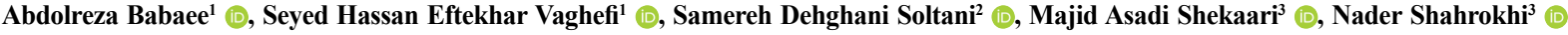
Mohsen Basiri ${ }^{3 *}$

1. Department of Anatomy, Afzalipour School of Medicine, Kerman University of Medical Sciences, Kerman, Iran.

2. Department of Anatomy, School of Medicine, Rafsanjan University of Medical Sciences, Rafsanjan, Iran.

3. Neuroscience Research Center, Institute of Neuropharmacology, Kerman University of Medical Sciences, Kerman, Iran

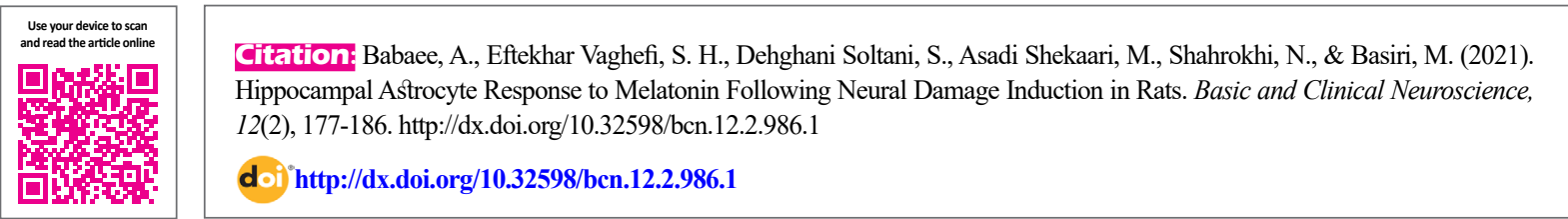

\section{(c) (i) (5)}

Article info:

Received: 28 Aug 2019

First Revision: 13 Sep 2019

Accepted: 18 May 2020

Available Online: 01 Mar 2021

Keywords:

Melatonin, Astrocyte, GFAP, Hippocampus

\section{A B S T RA C T}

Introduction: Brain injury induces an almost immediate response from glial cells, especially astrocytes. Activation of astrocytes leads to the production of inflammatory cytokines and reactive oxygen species that may result in secondary neuronal damage. Melatonin is an antiinflammatory and antioxidant agent, and it has been reported to exert neuroprotection through the prevention of neuronal death in several models of central nervous system injury. This study aimed to investigate the effect of melatonin on astrocyte activation induced by Traumatic Brain Injury (TBI) in rat hippocampus and dentate gyrus.

Methods: Animals were randomly divided into 5 groups; Sham group, TBI group, vehicle group, and melatonin-treated TBI groups (TBI+Mel5, TBI+Mel20). Immunohistochemical method (GFAP marker) and TUNEL assay were used to evaluate astrocyte reactivity and neuronal death, respectively.

Results: The results demonstrated that the astrocyte number was reduced significantly in melatonin-treated groups compared to the vehicle group. Additionally, based on TUNEL results, melatonin administration noticeably reduced the number of apoptotic neurons in the rat hippocampus and dentate gyrus.

Conclusion: In general, our findings suggest that melatonin treatment after brain injury reduces astrocyte reactivity as well as neuronal cell apoptosis in rat hippocampus and dentate gyrus. 


\section{Highlights}

- Melatonin markedly diminished astrocyte reactivity following traumatic brain injury.

- Melatonin treatment reduced the number of apoptotic neurons.

- Melatonin reduces astrocyte reactivity and cell death in a dose-independent pattern.

Plain Language Summary

Annually, many people lose their lives due to brain injury worldwide. Brain injury induces an almost immediate response from glial cells. Also, activation of glial cells may result in secondary neuronal damage. Melatonin is known as an anti-inflammatory agent, and it has been reported to exert neuroprotection.

\section{Introduction}

entral nervous system injury induces an almost immediate reaction in glial cells, especially astrocytes (Pekny \& Nilsson, 2005; Pineau, Sun, Bastien, \& Lacroix, 2010). Astrocytes contribute to brain homeostasis and comprise approximately $90 \%$ of total brain mass (Gee \& Keller, 2005). Previous studies have shown that primary neuronal injury caused an increase in reactive oxygen species production and inflammatory cytokines released by reactive astrocytes. These conditions may lead to secondary neuronal damage (Asadi-Shekaari, Basin, \& Babaee, 2014; Blasko et al., 2004; Ozdemir et al., 2005). The Cornu Ammonis (CA1) region of the hippocampus and neurons in the dentate gyrus (Farina, Aloisi, \& Meinl, 2007) are vulnerable to a variety of chemical, physical, and cytotoxic insults (Ansari, Roberts, \& Scheff, 2008; Barha, Ishrat, Epp, Galea, \& Stein, 2011; Liang et al., 2008). To diminish as much as possible the secondary neurological consequences following injuries of brain tissue, neuroprotective strategies such as using antioxidant compounds are urgently required (Hall,Vaishnav, \& Mustafa, 2010).

Melatonin is a tryptophan derivative that is synthesized generally in the pineal gland. This neurohormone controls the circadian rhythm and sleep induction (Hickie \& Rogers, 2011; Reiter, Tan, Osuna, \& Gitto, 2000). Also, melatonin has a better antioxidant and anti-inflammatory effect than vitamin $\mathrm{E}$ and can secondarily upsurge the expression of other antioxidant enzymes (Babaei-Balderlou, Zare, Heydari, \& Farokhi, 2010; Boutin, Audinot, Ferry, \& Delagrange, 2005). A recent study has shown that melatonin secretion decreased following neuronal injury (Seifman et al., 2014). Therefore, some research- ers, using exogenous administration of melatonin, examined its neuroprotective effects on several models of neural cell injuries (Ding et al., 2014; Dong et al., 2016). For instance, melatonin administration after neonatal hypoxic ischemia reduced neural cell death and reactive astrogliosis (Alonso-Alconada, Alvarez, Lacalle, \& Hilario, 2012). Brain injury research until quite recently has focused on the pathophysiology of injured neurons, while very little attention has been paid to non-neuronal cells (Barreto, Gonzalez, Torres, \& Morales, 2011; Burda, Bernstein, \& Sofroniew, 2016).

Because of inadequate attention to the melatonin effects on astrocytes reactivity and vulnerability of nervous tissue to inflammation and oxidative stresses (Leszek, Barreto, Gasiorowski, Koutsouraki, \& Aliev, 2016), we investigate the effects of exogenous administration of melatonin on astrocyte activation induced by traumatic brain injury in rat hippocampus and dentate gyrus.

\section{Methods}

\subsection{Animals and experimental protocols}

All experiments were done in accordance with the rules of the Ethics Committee of Medical Faculty (EC/ KNRC/90-2; Kerman University, Iran). A total of 35 male rats (NMRI, 230 to $275 \mathrm{~g}$ ) were kept in the animal cage with free access to food and water. Animals were randomly divided into 5 groups before Traumatic Brain Injury (TBI) induction: Sham (intact) $(n=7)$, TBI group $(n=7)$, vehicle $(n=7)$ that exposed to TBI and received an intraperitoneal injection of melatonin vehicle (ethanol + normal saline; $0.33 \mathrm{~mL} / \mathrm{rat}$ ) (Chern, Liao, Wang, \& Shen, 2012; Dehghan, Hadad, Asadikram, Najafipour, \& Shahrokhi 2013), and two TBI+melatonin groups that 
were exposed to TBI and then received $5 \mathrm{mg} / \mathrm{kg}$ and 20 $\mathrm{mg} / \mathrm{kg}$ melatonin (Sigma, St. Louis, MO), respectively at 1 hour, and then 1, 2 and 3 days post-TBI (Babaee et al., 2015; Gutierrez-Cuesta et al., 2007).

\subsection{Induction of TBI}

After incision in the skull skin, the animals were exposed to diffuse brain trauma through the Marmarou method (Marmarou et al., 1994). In this method, the skull of animals was enclosed with a metallic disc (with $3 \mathrm{~mm}$ thick and a diameter of $10 \mathrm{~mm}$ ), and then a $250 \mathrm{~g}$ weight (at the height of $2 \mathrm{~m}$ ) was thrown onto the skull of the rats. Following recovery, the rats were returned to their cages (Babaee et al., 2015; Marmarou et al., 1994).

\subsection{Preparing the brain tissue}

For histological evaluation, all rats were anesthetized with IP injection of $400 \mathrm{mg} / \mathrm{kg}$ chloral hydrate (Merck, 102425) (Mortezazadeh et al., 2018) $72 \mathrm{~h}$ after brain trauma and were perfused intracardially with 140-180 mL of heparinized $0.9 \%$ saline, followed by 100 to $120 \mathrm{~mL}$ of $4 \%$ paraformaldehyde in Phosphate-Buffered Saline (PBS). The perfusion continued until the lungs and liver were clear of blood (for 10 to $15 \mathrm{~min}$ ) (Babaee et al., 2019; Pourhoseini et al., 2017). Then, animals' brains were cautiously removed and immediately transferred in $10 \%$ formaldehyde and maintained overnight at $4^{\circ} \mathrm{C}$. The brains were then dehydrated by alcohol solution and finally embedded in paraffin (Babaee et al., 2015; Ding et al., 2014).

\subsection{TUNEL staining}

TUNEL staining was carried out on the cerebral sections using the apoptosis detection kit (Indianapolis, Roche, IN). Staining of sections was performed according to the manufacturer's instructions. Afterward, cerebral sections were incubated with DAB (3, 3'-diaminobenzidine) and hydrogen peroxide; the brownish color shows damage to neuronal perikarya (Hakemi, Sharififar, Haghpanah, Babaee, \& Eftekhar-Vaghefi, 2019; Varshosaz, Taymouri, Pardakhty, Asadi-Shekaari, \& Babaee, 2014). Also, negative and positive controls were included.

\subsection{Immunohistochemistry}

For assessing astrocytic reactivity, we used mouse monoclonal anti-GFAP (Glial Fibrillary Acidic Protein) antibody (Dako A/S Denmark, 1:400) (DehghaniSoltani, Shojaee, Jalalkamali, Babaee, \& NematollahiMahani, 2017). For this purpose, after deparaffinization in a microwave oven with $65^{\circ} \mathrm{C}$ and rinsing of sections in xylene (5 min), they washed with PBS and were put in the $10 \mathrm{mM}$ citrate buffer for $1 \mathrm{~h}$ (temperature of $90^{\circ} \mathrm{C}$ and $\mathrm{pH}$ 6). Then the sections were placed in $0.3 \% \mathrm{H} 2 \mathrm{O} 2$ for 12 minutes. After overnight incubation with primary antibody, the sections were washed with PBS and then incubated with secondary antibody (1:350, rabbit antimouse) for 2 hours at room temperature (Babaee et al., 2015; Babaee et al., 2018).

\subsection{Cell counting}

Five coronal sections of the hippocampus were chosen for cell counting. In each section, the TUNEL positive cells were counted in four fields of dentate gyrus and hippocampus at 200× magnification (Pazar et al., 2016). Additionally, GFAP positive cells (astrocytes) were counted in four random and non-overlapping regions (Eftekhar-Vaghefi, Raygan, Eftekhar-Vaghefi, Dehghani-Soltani, \& Babaee, 2017; Soltani et al., 2016) of the hippocampus and dentate gyrus using an optical microscope (TS100, Nikon, Japan).

\subsection{Statistical analysis}

The obtained data were expressed as Mean \pm SEM. One-way ANOVA followed by Tukey-Kramer multiple post hoc test was used to assess the significant differences $(\mathrm{P}<0.05)$ between groups (Mortazavi, 2009).

\section{Results}

After preparation of coronal sections, including the hippocampus and dentate gyrus, some of them were stained with hematoxylin and eosin for morphological evaluation (Figure 1), and the others were subjected to TUNEL staining and immunohistochemistry.

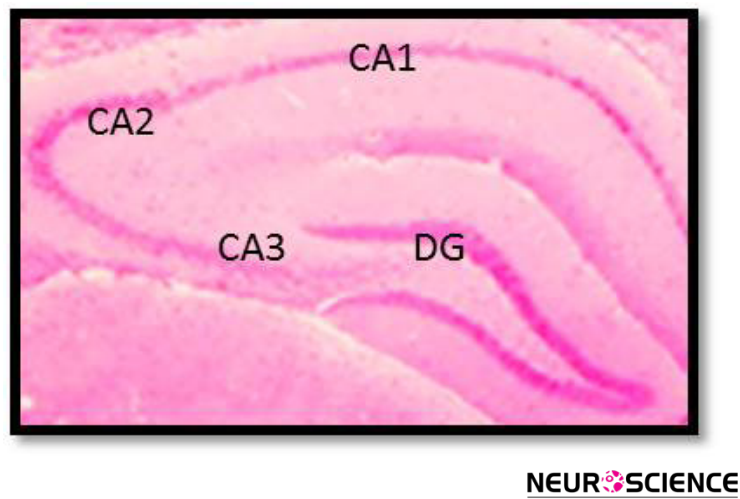

Figure 1. A coronal section of the rat brain that indicated the cornu Ammonis regions (CA1, CA2, CA3) of the hippocampus as well as Dentate Gyrus (DG) (H\&E staining, 100×). 

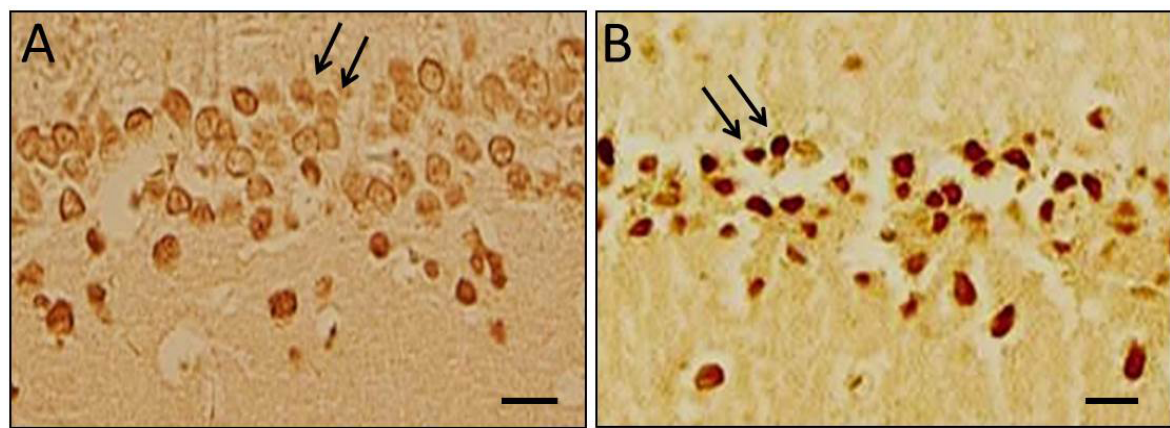

NEUR SCIENCE

Figure 2. Neuronal apoptosis

Control group (A): Arrows showing the healthy neurons; TBI group (B): Arrows showing the TUNEL positive cells (apoptotic neurons); Scale bar $=15 \mu \mathrm{m}$.

\subsection{Evaluation of apoptotic cells}

Our findings showed that apoptotic cells were significantly increased $(\mathrm{P}<0.001)$ in dentate gyri and hippocampi of TBI group rats in comparison to the sham group, while the number of apoptotic neurons in the melatonin-treated groups was significantly decreased (Figures 2 and 3) that were not dose-dependent.

\subsection{The number of activated astrocytes}

In the present study, increased GFAP-positive astrocytes were detected in the hippocampi and dentate gyri of TBI group rats compared to that in the sham rats (Figures 4 and 5). Also, treatment with melatonin significantly $(\mathrm{P}<0.01)$ reduced activated astrocytes that were not dose-dependent (Figure 6).

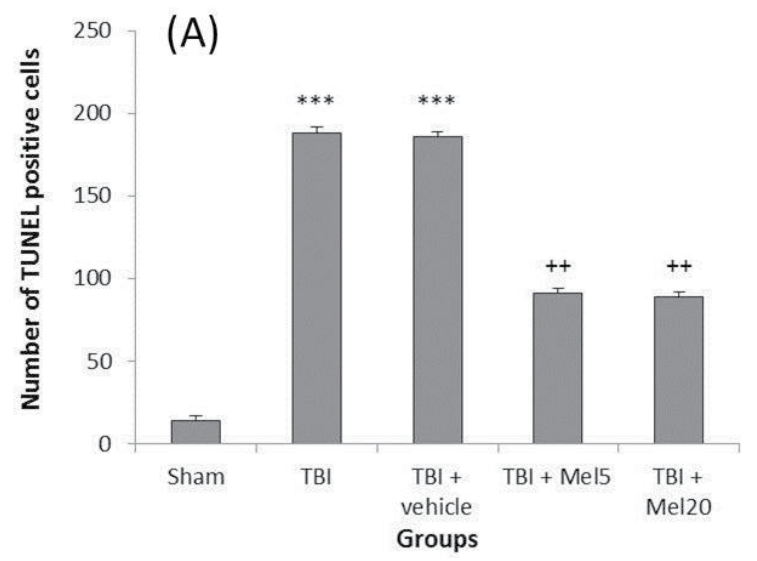

\section{Discussion}

The results of the current study have demonstrated the neuroprotective effect of melatonin after traumatic injury in rats. Acquired data indicate that TBI leads to a significant increase in neuronal cell death in the hippocampus and dentate gyrus, and based on TUNEL staining, the number of apoptotic cells noticeably decreased in the melatonin treatment groups (Mel5 and Mel20). Gao et al. reported that traumatic brain injury causes synaptic and dendritic degeneration in the dentate gyrus (Gao, Deng, $\mathrm{Xu}, \&$ Chen, 2011). Also, Hung et al. have shown that melatonin alleviates hippocampal injury following hypoxia (Hung, Tipoe, Poon, Reiter, \& Fung, 2008). Annually, more than 1 million people die following TBI worldwide (Babaee et al., 2015). Neural inflammation and production of oxidative stress are two major pathological mechanisms of neuronal cell death after TBI (Cornelius et al., 2013; Woodcock \& Morganti-Koss-

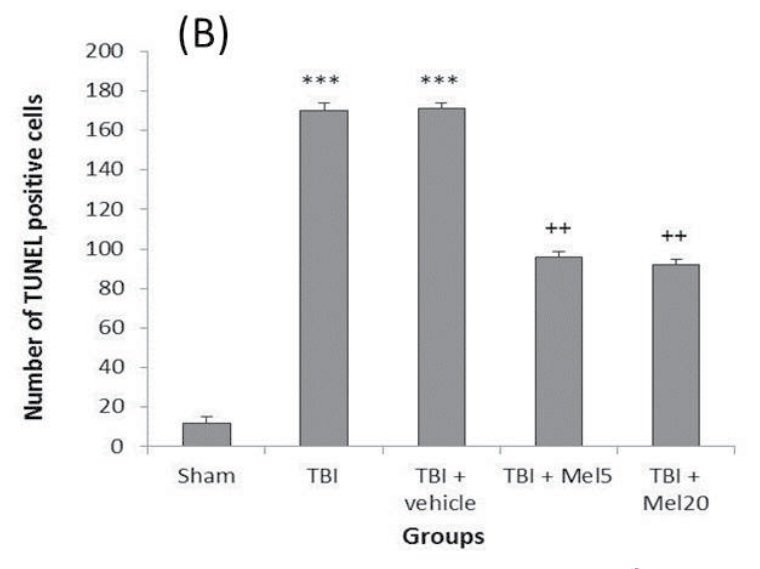

Figure 3. Melatonin treatment reduced apoptosis that induced by TBI in hippocampi (A) and dentate gyri (B) of rats

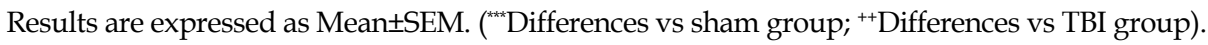




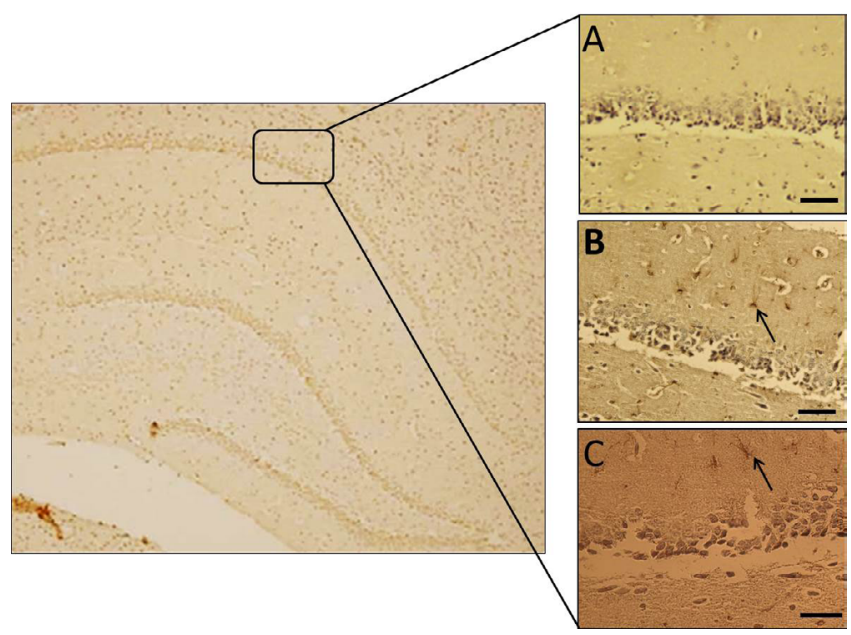

Figure 4. Immunohistochemical analysis of GFAP-positive cells

Hippocampal astrocytes in Sham group (A): TBI group; and (B): TBI+Mel20 group; Arrows display the astrocytes (Scale bar=35 $\mu \mathrm{m})$.

mann, 2013). To inhibit secondary injuries after primary brain injury, it is essential to limit the neural inflammation via decreasing the astrocyte activation (Kabadi, Stoica, Loane, Luo, \& Faden 2014). Chern et al. have shown that intraperitoneal administration of melatonin improved the neuronal survival rate in ischemic-stroke mice (Chern et al., 2012).

Besides, melatonin has been assessed as an effective medicine in TBI through increasing glutathione peroxidase and superoxide dismutase activities (Dehghan et al., 2013). The result of this study does not show any noticeable difference between two different doses of melatonin. Ozdemir et al. reported that melatonin significantly reduced oxidative damage induced by TBI in immature rats, which was equally effective at different doses of 5 $\mathrm{mg} / \mathrm{kg}$ and $20 \mathrm{mg} / \mathrm{kg}$ (Ozdemir et al., 2005).
Glial cells activation, particularly astrocytes, occurs in response to different injuries of brain tissue, such as trauma, chemical injuries, tumor formation, brain ischemia, and neurodegenerative disease (Guo et al., 2014; Hald, Nedergaard, Hansen, Ding, \& Heegaard, 2009; Lee et al., 2010). Activation of astrocytes following brain injury has known as astrogliosis. Previous studies have demonstrated that up-regulation of GFAP occurs during the astrogliosis phenomenon (Hostenbach, Cambron, D'haeseleer, Kooijman, \& De Keyser, 2014; Kamphuis et al., 2012). Therefore, this study has focused on astrocyte activation based on GFAP immunoreactivity.

Astrocytes, as the most abundant glial cells in brain tissue (Farina et al., 2007), maybe the target of melatonin. Our findings have shown that the number of astrocytes is decreased in the melatonin treatment groups, which shows the alleviation of astrogliosis induced by TBI. In

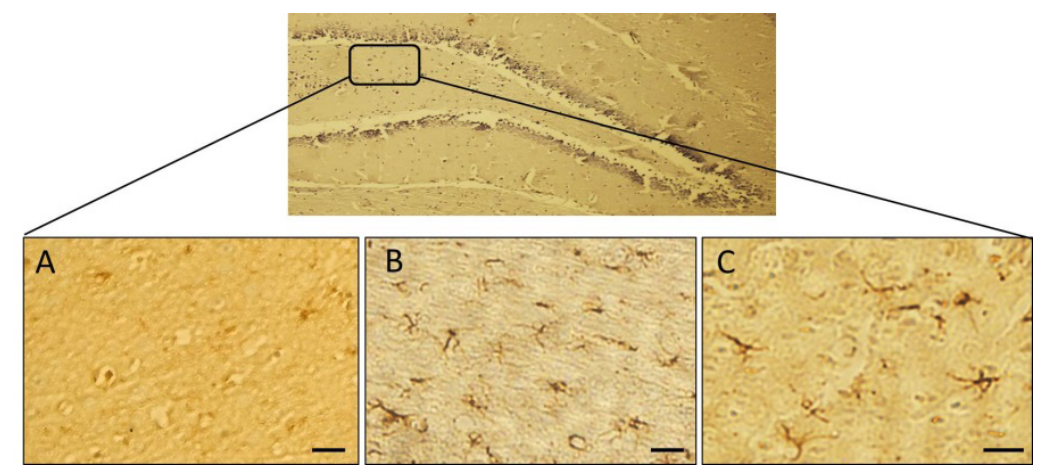

Figure 5. Immunohistochemical analysis of GFAP-positive cells in the dentate gyrus of different groups

A: Sham group; B: TBI group; and TBI + Mel20 group (Scale bar=15 $\mu \mathrm{m})$. 

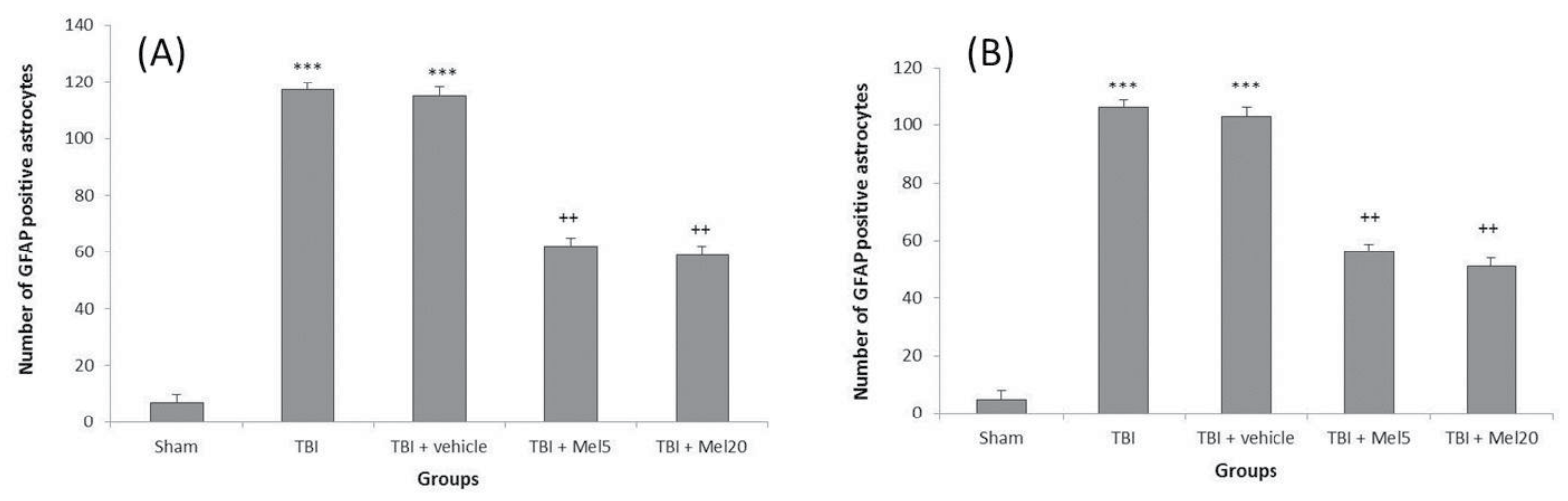

Figure 6. Melatonin treatment

NEUR SCIENCE

A: Decreased astrocytes in hippocampi; and B: Dentate gyri of rats following TBI induction. The results are presented as Mean \pm SEM ("it Differences vs sham group; ${ }^{++}$Differences vs TBI group).

an experimental study, Ananth et al. demonstrated that domoic acid-induced astrogliosis is attenuated significantly in the hippocampus of adult rats using exogenous administration of melatonin (Ananth, Gopalakrishnakone, \& Kaur, 2003). Barreto et al. reported that astrocytic-neuronal interactions could act as a neuroprotective strategy against brain injury (Barreto et al., 2011).

The blood brain barrier has an important role in exacerbating neuronal damage following traumatic brain injury (Abbott, Patabendige, Dolman, Yusof, \& Begley, 2010; Persidsky, Ramirez, Haorah, \& Kanmogne, 2006). In other words, damage of this barrier resulted in entrance of neutrophils, lymphocytes and monocytes to the injured site. Glial cells are then activated and induce inflammation that may promote neuronal death (Seo et al., 2013; Ziebell \& Morganti-Kossmann, 2010).

In addition, Tsai et al. demonstrated that melatonin administration reduced proinflammatory cytokines via upregulation of STAT1 DNA binding activity (Tsai, Chen, Tsai, Ching, \& Chuang, 2011). However, the modulation actions of melatonin to astrocyte, which was introduced as the primary source of proinflammatory cytokines such as IL-6 $\beta$, have not yet been widely examined. Our experiment only shows the neuroprotective effects of melatonin following a short period after brain injury $(1 \mathrm{~h}$ to $72 \mathrm{~h}$ ). Thus, additional investigations need to be done to elucidate the molecular mechanism of melatonin to alleviate astrocyte reactivity.

\section{Ethical Considerations}

\section{Compliance with ethical guidelines}

Animal procedures in this research were done in compliance with the Guide for the Care and Use of Laboratory Animals and certified by the local Animal Ethics Committee of Kerman University of Medical Sciences (Kerman, Iran) in 2016 (EC/KNRC/90-2).

\section{Funding}

This study was extracted from the MSc. thesis of Abdolreza Babaee, and financially supported by Neuroscience Research Center, Institute of Neuropharmacology, Kerman University of Medical Sciences, Kerman, Iran (EC/KNRC/90-2).

\section{Authors' contributions}

Conceptualization, data collection, and writing original draft: Abdolreza Babaee, Samereh Dehghani-Soltani; Data analysis, writing - review \& editing, investigation, supervision: Seyed Hassan Eftekhar-Vaghefi Majid Asadi-Shekaari, Nader Shahrokhi, Mohsen Basiri.

\section{Conflict of interest}

The authors declared no conflict of interest.

\section{Acknowledgments}

We would like to thank the personnel of the Department of Anatomy, Kerman University of Medical Sciences, Kerman, for their cooperation. 


\section{References}

Abbott, N. J., Patabendige, A. A., Dolman, D. E., Yusof, S. R., \& Begley, D. J. (2010). Structure and function of the blood-brain barrier. Neurobiology of Disease, 37(1), 13-25. [DOI:10.1016/j. nbd.2009.07.030] [PMID]

Alonso-Alconada, D., Alvarez, A., Lacalle, J., \& Hilario, E. (2012) Histological study of the protective effect of melatonin on neural cells after neonatal hypoxia-ischemia. Histology and Histopathology, 27(6), 771-83. http://hdl.handle.net/10201/52455

Ananth, C., Gopalakrishnakone, P., \& Kaur, C. (2003). Protective role of melatonin in domoic acid-induced neuronal damage in the hippocampus of adult rats. Hippocampus, 13(3), 375-87. [DOI:10.1002/ hipo.10090] [PMID]

Ananth, C., Gopalakrishnakone, P., \& Kaur, C. (2003). Protective role of melatonin in domoic acid-induced neuronal damage in the hippocampus of adult rats. Hippocampus, 13(3), 375-87. [DOI:10.1016/j.freeradbiomed.2008.04.038] [PMID] [PMCID]

Ansari, M. A., Roberts, K. N., \& Scheff, S. W. (2008). Oxidative stress and modification of synaptic proteins in hippocampus after traumatic brain injury. Free Radical Biology and Medicine, 45(4), 443-52. [DOI:10.1016/j.freeradbiomed.2008.04.038]

Asadi-Shekaari, M., Basin, M., \& Babaee, A. (2014). The protective effects of walnuts (Juglans regia) on neuronal death and astrocyte reactivity following middle cerebral artery occlusion in male rats. Scientific Journal of Kurdistan University of Medical Sciences, 19(4), 100-8. https://www.cabdirect.org/cabdirect/abstract/20153067335

Babaee, A., Eftekhar-Vaghefi, S. H., Asadi-Shekaari, M., Shahrokhi, N., Soltani, S. D., \& Malekpour-Afshar, R., et al. (2015). Melatonin treatment reduces astrogliosis and apoptosis in rats with traumatic brain injury. Iranian journal of basic medical sciences, 18(9), 867. https://www.ncbi.nlm.nih.gov/pmc/articles/PMC4620185/

Babaee, A., Nematollahi-Mahani, S. N., Dehghani-Soltani, S., Shojaei, M., \& Ezzatabadipour, M. (2019). Photobiomodulation and gametogenic potential of human Wharton's jelly-derived mesenchymal cells. Biochemical and Biophysical Research Communications, 514(1), 239-45. [DOI:10.1016/j.bbrc.2019.04.059] [PMID]

Babaee, A., Nematollahi-Mahani, S. N., Shojaei, M., DehghaniSoltani, S., \& Ezzatabadipour, M. (2018). Effects of polarized and non-polarized red-light irradiation on proliferation of human Wharton's jelly-derived mesenchymal cells. Biochemical and Biophysical Research Communications, 504(4), 871-7.[DOI:10.1016/j. bbrc.2018.09.010] [PMID]

Babaei, B. F., Zare, S., Heydari, R., \& Farokhi, F. (2010). Effects of melatonin and vitamin $E$ on peripheral neuropathic pain in streptozotocin-induced diabetic rats. Iranian Journal of Basic Medical Sciences, 13(2), 1-8. https://www.sid.ir/en/journal/ViewPaper. aspx?ID=168971

Barha, C. K., Ishrat, T., Epp, J. R., Galea, L. A., \& Stein, D. G. (2011) Progesterone treatment normalizes the levels of cell proliferation and cell death in the dentate gyrus of the hippocampus after traumatic brain injury. Experimental Neurology, 231(1), 72-81. [DOI:10.1016/j.expneurol.2011.05.016] [PMID] [PMCID]

Barreto, G. E., Gonzalez, J., Torres, Y., \& Morales, L. (2011). Astrocytic-neuronal crosstalk: Implications for neuroprotection from brain injury. Neuroscience Research, 71(2), 107-13. [DOI:10.1016/j. neures.2011.06.004] [PMID]
Blasko, I., Stampfer-Kountchev, M., Robatscher, P., Veerhuis, R Eikelenboom, P., \& Grubeck-Loebenstein, B. (2004). How chronic inflammation can affect the brain and support the development of Alzheimer's disease in old age: The role of microglia and astrocytes. Aging Cell, 3(4), 169-76. [DOI:10.1111/j.14749728.2004.00101.x] [PMID]

Boutin, J. A., Audinot, V., Ferry, G., \& Delagrange, P. (2005). Molecular tools to study melatonin pathways and actions. Trends in Phar macological Sciences, 26(8), 412-9. [DOI:10.1016/j.tips.2005.06.006] [PMID]

Burda, J. E., Bernstein, A. M., \& Sofroniew, M. V. (2016). Astrocyte roles in traumatic brain injury. Experimental Neurology, 275, 305-15. [DOI:10.1016/j.expneurol.2015.03.020] [PMID] [PMCID]

Chern, C. M., Liao, J. F., Wang, Y. H., \& Shen, Y. C. (2012). Melatonin ameliorates neural function by promoting endogenous neurogenesis through the MT2 melatonin receptor in ischemic-stroke mice Free Radical Biology and Medicine, 52(9), 1634-47. [DOI:10.1016/j. freeradbiomed.2012.01.030] [PMID]

Cornelius, C., Crupi, R., Calabrese, V., Graziano, A., Milone, P., \& Pennisi, G., et al. (2013). Traumatic brain injury: Oxidative stress and neuroprotection. Antioxidants \& Redox Signaling, 19(8), 836-53. [DOI:10.1089/ars.2012.4981] [PMID]

Dehghan, F., Hadad, M. K., Asadikram, G., Najafipour, H., \& Shahrokhi, N. (2013). Effect of melatonin on intracranial pressure and brain edema following traumatic brain injury: Role of oxidative stresses. Archives of Medical Research, 44(4), 251-8. [DOI:10.1016/j. arcmed.2013.04.002] [PMID]

Dehghani-Soltani, S., Shojaee, M., Jalalkamali, M., Babaee, A., \& Nematollahi-Mahani, S. N. (2017). Effects of light emitting diode irradiation on neural differentiation of human umbilical cord-derived mesenchymal cells. Scientific Reports, 7(1), 1-9.[DOI:10.1038/ s41598-017-10655-w] [PMID] [PMCID]

Ding, K., Wang, H., Xu, J., Li, T., Zhang, L., \& Ding, Y., et al. (2014). Melatonin stimulates antioxidant enzymes and reduces oxidative stress in experimental traumatic brain injury: The Nrf2-ARE signaling pathway as a potential mechanism. Free Radical Biology and Medicine, 73, 1-11. [DOI:10.1016/j.freeradbiomed.2014.04.031] [PMID]

Dong, Y., Fan, C., Hu, W., Jiang, S., Ma, Z., \& Yan, X., et al. (2016) Melatonin attenuated early brain injury induced by subarachnoid hemorrhage via regulating NLRP 3 inflammasome and apoptosis signaling. Journal of Pineal Research, 60(3), 253-62. [DOI:10.1111/ jpi.12300] [PMID]

Eftekhar-Vaghefi, S. H., Raygan, S. P., Eftekhar-Vaghefi, S., Dehghani-Soltani, S., \& Babaee, A. (2017). An evaluation of tissue destruction time in phrenic nerve after death and counting the number of nerve fibers in C3, C4 and C5 branches. Journal of Babol University of Medical Sciences, 19(3), 59-65. [DOI:10.22088/jbums.19.3.59]

Farina, C., Aloisi, F., \& Meinl, E. (2007). Astrocytes are active players in cerebral innate immunity. Trends in Immunology, 28(3), 138-45. [DOI:10.1016/j.it.2007.01.005] [PMID]

Gao, X., Deng, P., Xu, Z. C., \& Chen, J. (2011). Moderate traumatic brain injury causes acute dendritic and synaptic degeneration in the hippocampal dentate gyrus. PloS One, 6(9), e24566. [DOI:10.1371/journal.pone.0024566] [PMID] [PMCID]

Gee, J. R., \& Keller, J. N. (2005). Astrocytes: Regulation of brain homeostasis via apolipoprotein E. The International Journal of Biochemistry \& Cell Biology, 37(6), 1145-50. [DOI:10.1016/j.biocel.2004.10.004] [PMID] 
Guo, Z., Zhang, L., Wu, Z., Chen, Y., Wang, F., \& Chen, G. (2014). In vivo direct reprogramming of reactive glial cells into functional neurons after brain injury and in an Alzheimer's disease model. Cell Stem Cell, 14(2), 188-202. [DOI:10.1016/j. stem.2013.12.001] [PMID] [PMCID]

Gutierrez-Cuesta, J., Sureda, F. X., Romeu, M., Canudas, A. M., Caballero, B., \& Coto-Montes, A., et al. (2007). Chronic administration of melatonin reduces cerebral injury biomarkers in SAMP8. Journal of Pineal Research, 42(4), 394-402. [DOI:10.1111/j.1600-079X.2007.00433.x] [PMID]

Hakemi, S. G., Sharififar, F., Haghpanah, T., Babaee, A., \& Eftekhar-Vaghefi, S. H. (2019). The effects of olive leaf extract on the testis, sperm quality and testicular germ cell apoptosis in male rats exposed to busulfan. International Journal of Fertility \& Sterility, 13(1), 57-65. https://www.ncbi.nlm.nih.gov/ pmc/articles/PMC6334023/

Hald, A., Nedergaard, S., Hansen, R. R., Ding, M., \& Heegaard, A. M. (2009). Differential activation of spinal cord glial cells in murine models of neuropathic and cancer pain. European Journal of Pain, 13(2), 138-45. [DOI:10.1016/j.ejpain.2008.03.014] [PMID]

Hall, E. D., Vaishnav, R. A., \& Mustafa, A. G. (2010). Antioxidant therapies for traumatic brain injury. Neurotherapeutics, 7(1), 51-61. [DOI:10.1016/j.nurt.2009.10.021] [PMID] [PMCID]

Hickie, I. B., \& Rogers, N. L. (2011). Novel melatonin-based therapies: Potential advances in the treatment of major depression. The Lancet, 378(9791), 621-31. [DOI:10.1016/S01406736(11)60095-0]

Hostenbach, S., Cambron, M., D’haeseleer, M., Kooijman, R., \& De Keyser, J. (2014). Astrocyte loss and astrogliosis in neuroinflammatory disorders. Neuroscience Letters, 565, 39-41. [DOI:10.1016/j.neulet.2013.10.012] [PMID]

Hung, M. W., Tipoe, G. L., Poon, A. M. S., Reiter, R. J., \& Fung, M. L. (2008). Protective effect of melatonin against hippocampal injury of rats with intermittent hypoxia. Journal of Pineal Research, 44(2), 214-21. [DOI:10.1111/j.1600-079X.2007.00514.x] [PMID]

Kabadi, S. V., Stoica, B. A., Loane, D. J., Luo, T., \& Faden, A. I. (2014). CR8, a novel inhibitor of CDK, limits microglial activation, astrocytosis, neuronal loss, and neurologic dysfunction after experimental traumatic brain injury. Journal of Cerebral Blood Flow \& Metabolism, 34(3), 502-13. [DOI:10.1038/ jcbfm.2013.228] [PMID] [PMCID]

Kamphuis, W., Mamber, C., Moeton, M., Kooijman, L., Sluijs, J. A., \& Jansen, A. H., et al. (2012). GFAP isoforms in adult mouse brain with a focus on neurogenic astrocytes and reactive astrogliosis in mouse models of Alzheimer disease PloS One, 7(8), e42823. [DOI:10.1371/journal.pone.0042823] [PMID] [PMCID]

Lee, M., Cho, T., Jantaratnotai, N., Wang, Y. T., McGeer, E., \& McGeer, P. L. (2010). Depletion of GSH in glial cells induces neurotoxicity: Relevance to aging and degenerative neurological diseases. The FASEB Journal, 24(7), 2533-45. [DOI:10.1096/ fj.09-149997] [PMID]

Leszek, J., E Barreto, G., Gasiorowski, K., Koutsouraki, E., \& Aliev, G. (2016). Inflammatory mechanisms and oxidative stress as key factors responsible for progression of neurodegeneration: Role of brain innate immune system. CNS E Neurological Disorders-Drug Targets (Formerly Current Drug Targets-CNS \& Neurological Disorders), 15(3), 329-36. [DOI:10. 2174/1871527315666160202125914] [PMID]
Liang, H. W., Qiu, S. F., Shen, J., Sun, L. N., Wang, J. Y., \& Bruce, I. C., et al. (2008). Genistein attenuates oxidative stress and neuronal damage following transient global cerebral ischemia in rat hippocampus. Neuroscience Letters, 438(1), 116-20. [DOI:10.1016/j.neulet.2008.04.058] [PMID]

Marmarou, A., Foda, M. A. A. E., Van Den Brink, W., Campbell, J., Kita, H., \& Demetriadou, K. (1994). A new model of diffuse brain injury in rats: Part I: Pathophysiology and biomechanics. Journal of Neurosurgery, 80(2), 291-300. [DOI:10.3171/ jns.1994.80.2.0291] [PMID]

Mortezazadeh, F., Fathabady, F. F., Norouzian, M., Noureddin, S., Rouholamini, S. E. Y., \& Babaee, A., et al. (2018). Investigating the effect of tumor necrosis factor Alpha on placenta and gene related bone formation of newborn mice. Journal of Research in Medical and Dental Science, 6(5), 133-8. https:/ / www.researchgate.net/profile/Mohsen-Basiri/publication/331311088

Ozdemir, D., Uysal, N., Gonenc, S., Acikgoz, O., Sonmez, A., \& Topcu, A., et al. (2005). Effect of melatonin on brain oxidative damage induced by traumatic brain injury in immature rats Physiological Research, 54(6), 631. https://www.researchgate. net/profile/Osman-Acikgoz/publication/8013574_

Pazar, A., Kolgazi, M., Memisoglu, A., Bahadir, E., Sirvanci, S., \& Yaman, A., et al. (2016). The neuroprotective and anti-apoptotic effects of melatonin on hemolytic hyperbilirubinemiainduced oxidative brain damage. Journal of Pineal Research, 60(1), 74-83. [DOI:10.1111/jpi.12292] [PMID]

Pekny, M., \& Nilsson, M. (2005). Astrocyte activation and reactive gliosis. Glia, 50(4), 427-34. [DOI:10.1002/glia.20207] [PMID]

Persidsky, Y., Ramirez, S. H., Haorah, J., \& Kanmogne, G. D. (2006). Blood-brain barrier: Structural components and function under physiologic and pathologic conditions. Journal of Neuroimmune Pharmacology, 1(3), 223-36. [DOI:10.1007/ s11481-006-9025-3] [PMID]

Pineau, I., Sun, L., Bastien, D., \& Lacroix, S. (2010). Astrocytes initiate inflammation in the injured mouse spinal cord by promoting the entry of neutrophils and inflammatory monocytes in an IL-1 receptor/MyD88-dependent fashion. Brain, Behavior, and Immunity, 24(4), 540-53. [DOI:10.1016/j. bbi.2009.11.007] [PMID]

Pourhoseini, S., Bakhtiari, M., Babaee, A., Ostovan, M. A., Eftekhar-Vaghefi, S. H., \& Ostovan, N., et al. (2017). Increased risk of coronary perforation during percutaneous intervention of myocardial bridge: What histopathology says Journal of Cardiovascular and Thoracic Research, 9(2), 108-12. [DOI:10.15171/jcvtr.2017.18] [PMID] [PMCID]

Reiter, R. J., Tan, D. X., Osuna, C., \& Gitto, E. (2000). Actions of melatonin in the reduction of oxidative stress. Journal of Biomedical Science, 7(6), 444-58. [DOI:10.1007/BF02253360] [PMID]

Seifman, M. A., Gomes, K., Nguyen, P. N., Bailey, M., Rosenfeld, J. V., \& Cooper, D. J., et al. (2014). Measurement of serum melatonin in intensive care unit patients: Changes in traumatic brain injury, trauma, and medical conditions. Frontiers in Neurology, 5, 237. [DOI:10.3389/fneur.2014.00237] [PMID] [PMCID]

Seo, J. H., Miyamoto, N., Hayakawa, K., Pham, L. D. D., Maki, T., \& Ayata, C., et al. (2013). Oligodendrocyte precursors induce early blood-brain barrier opening after white matter injury. The Journal of Clinical Investigation, 123(2), 782-6. [DOI:10.1172/JCI65863] [PMID] [PMCID] 
Mortavazi, S., Habib, A., Ganj-Karami, A., Samimi-Doost, R., Pour-Abedi, A., \& Babaie, A. (2009). Alterations in TSH and thyroid hormones following mobile phone use. Oman Medical Journal, 24(4), 274-300. [PMCID] [PMID]

Soltani, S. D., Babaee, A., Shojaei, M., Salehinejad, P., Seyedi, F., \& JalalKamali, M., et al. (2016). Different effects of energy dependent irradiation of red and green lights on proliferation of human umbilical cord matrix-derived mesenchymal cells. Lasers in Medical Science, 31(2), 255-61. [DOI:10.1007/s10103015-1846-y] [PMID]

Tsai, M. C., Chen, W. J., Tsai, M. S., Ching, C. H., \& Chuang, J. I. (2011). Melatonin attenuates brain contusion-induced oxidative insult, inactivation of signal transducers and activators of transcription 1, and upregulation of suppressor of cytokine signaling-3 in rats. Journal of Pineal Research, 51(2), 233-45. [DOI:10.1111/j.1600-079X.2011.00885.x] [PMID]

Varshosaz, J., Taymouri, S., Pardakhty, A., Asadi-Shekaari, M., \& Babaee, A. (2014). Niosomes of ascorbic acid and a-tocopherol in the cerebral ischemia-reperfusion model in male rats. $\mathrm{Bi}$ oMed Research International, 2014. [DOI:10.1155/2014/816103] [PMID] [PMCID]

Woodcock, T., \& Morganti-Kossmann, C. (2013). The role of markers of inflammation in traumatic brain injury. Frontiers in neurology, 4, 18. https://www.frontiersin.org/articles/10.3389/fneur.2013.00018/full

Ziebell, J. M., \& Morganti-Kossmann, M. C. (2010). Involvement of pro-and anti-inflammatory cytokines and chemokines in the pathophysiology of traumatic brain injury. Neurotherapeutics, 7(1), 22-30. [DOI:10.1016/j.nurt.2009.10.016] [PMID] [PMCID] 
This Page Intentionally Left Blank 